\title{
Indian Paradigm of Orthopedic Care in Post-COVID-19 Era
}

\author{
Madhan Jeyaraman ${ }^{1,2}$, Sathish Muthu ${ }^{1,2}$, Ashok Shyam ${ }^{1}$
}

\section{Abstract}

Introduction: COVID-19 pandemic caused by the newly emerged strain of coronavirus (SARS-CoV-2) has had phenomenally casted its impact on the health-care systems globally. The rampant spread of contagiosity has challenged the solidarity of the medical fraternity of the developed and developing world. The rising turmoil enforces to trudge with stoicism and expresses the need for planning because of subjugating the prevailing conditions with judicial channelization of available resources. In many developed and developing countries, the resources such as appropriate equipment as well as personnel have been extended to combat the pandemic substantially. At the same time, the populous country such as India has taken a stand to cancel electively planned orthopedic surgeries. However, under the issued guidelines of apex authorities, trauma and emergency services had have been in continuity with a reorganized manner. Hereby, we discuss the present shift in paradigm in the field of orthopedics with an interplay of regenerative orthopedics and telemedicine and its pivotal role against the odds of the COVID-19 pandemic. Besides, we see over the future perspectives and challenges in the purview of resorting to an effective clinical practice in orthopedics specialty. Albeit these guidelines expound strategies to manage trauma and orthopedic cases amidst pandemics but the subsequent post-COVID-19 phase warrants explicable vision and planning. Indeed, resuming elective orthopedics surgical intervention in post-phase of pandemic shall definitively be a task invoking fundamental planning, especially in a resource-limited background. With the rollout of vaccines in the country, the scenario is in favor of returning to normalcy with evaluation for COVID-19 being added to the list of routine medical and surgical screening profiles.

Keywords: COVID-19, SARS-CoV-2, pandemic, India, orthopedics.

\section{Introduction}

COVID-19/severe acute respiratory syndrome coronavirus-2 (SARS-CoV-2) broke out in Wuhan, China in December 2019, resulting in the rapid spread of severe respiratory illness across the country [1]. Within a few weeks, outbreaks of SARS-CoV-2 were recognized in several countries, especially Italy, France, Iran, and the USA. On March 11, 2020, the World Health Organization (WHO) declared COVID-19 as a pandemic affecting 100 countries with more than 100,000 cases [2]. Having tackled the first wave of the pandemic every nation is equipping itself with the current rollout of vaccines and establishing stringent standard operative protocols toward health-care practices. The current trend in the management of orthopedic patients in this post-COVID-19 era is depicted in
(Fig. 1).

\section{Stages of Orthopedic Practice in Post-COVID-19 Era}

a) Planning

The resumption of orthopedic practices in the post-COVID-19 era needs dynamic planning. The planning guidance has to be sought from apex bodies such as WHO, Centers for Disease Control and Prevention, Ministry of Health and Family Welfare (MOHFW), Indian Council of Medical Research (ICMR), and national organizations of microbiology, tropical medicine, infectious diseases, surgical specialties, and anesthesia, respectively. Interdisciplinary planning among all specialties helps to update the current protocol issued on changing trends on the pandemic condition and to mitigate the secondary waves ofCOVID-19[3, 4, 5, 6] (Fig. 2).
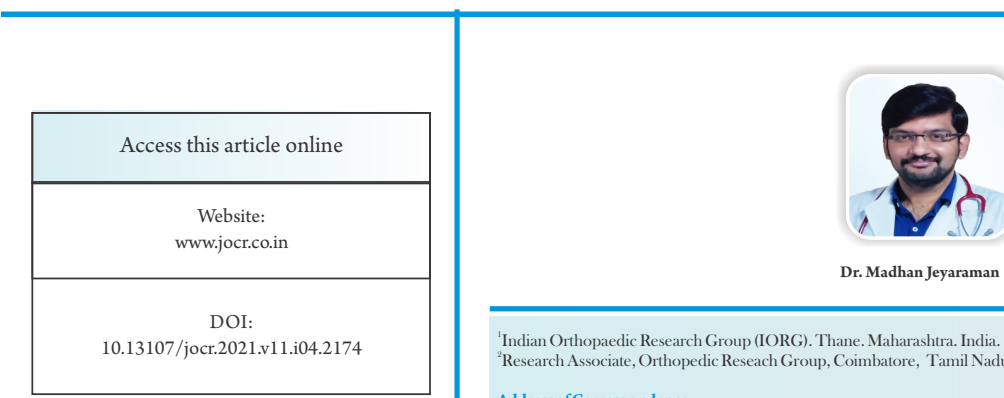

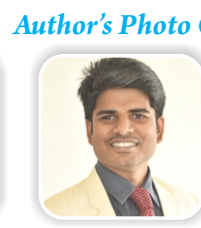

Dr. Sathish Muthu

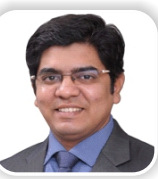

Dr. Ashok Shyam
'Indian Orthopaedic Research Group (IORG). Thane. Maharashtra. India.

"Research Associate, Orthopedic Reseach Group, Coimbatore, Tamil Nadu, India.

Address of Correspondence

Dr. Madhan Jeyaraman,
Indian Orthopaedic Research Group (IORG), Thane, Maharashtra, India

Email: madhanjeyaraman@gmail.com

Journal of Orthopaedic Case Reports | pISSN 2250-0685 | eISSN 2321-3817 | Available on www.jocr.co.in | doi:10.13107/jocr.2021.v11.i04.2174 This is an Open Access article distributed under the terms of the Creative Commons Attribution Non-Commercial License (http://creativecommons.org/licenses/by-nc/3.0) which permits unrestricted non-commercial use, distribution, and reproduction in any medium, provided the original work is properly cited. 


\section{Patient presenting with history of fracture/trauma/scheduled for elective surgical intervention at Orthopaedics Department}
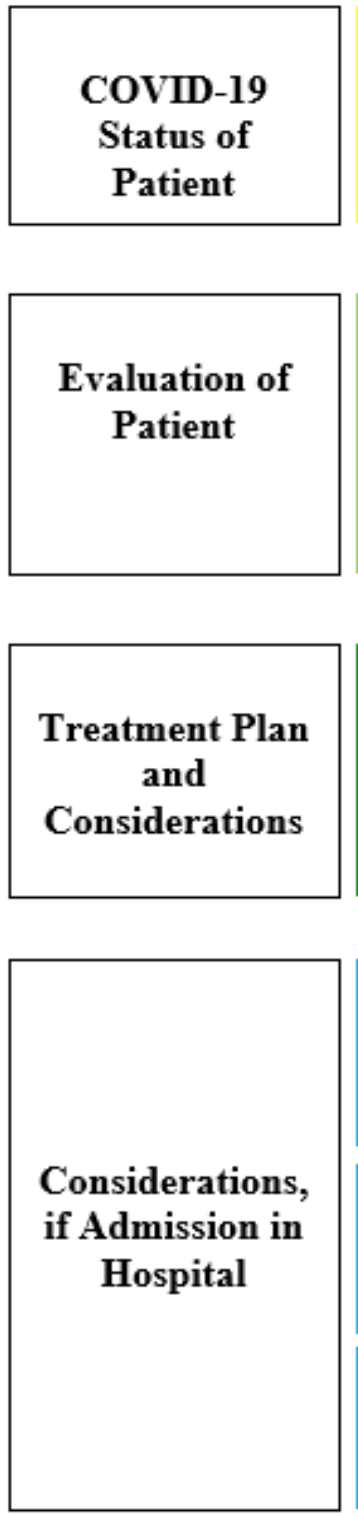

- Hospital Set-up: Triage principle on the basis of COVID-19 status of the patient; segregation of wards; adequate disinfection measures; adequate BMW management and regulation.

Evaluate the patient in concordance with patient's COVID-19 status.

- Immediate splintage and follow ATLS guidelines

- Stable (Clinical/Radiological findings to define best intervention)

- Unstable (ICU Care; temporary stabilization of fracture and delay surgical intervention)
- Emergency Surgery (to save life or salvage limb as in trauma cases)

- Damage Control Orthopaedics

- Non-operative principles and telemedicine

- Regenerative Orthopaedics
- Patient Oriented Aspects: Mandatory screening protocol for patient and attenders; minimal attenders; adequate hand hygiene and respiratory etiquettes.

- Health Personnel: Face shield and other protective measures to prevent cross infection; counsel and educating patients; multidisciplinary approach for better outcomes (as needed); research works under safety.

\begin{tabular}{|c|}
\hline $\begin{array}{c}\text { Follow up of } \\
\text { Patient }\end{array}$ \\
\hline
\end{tabular}

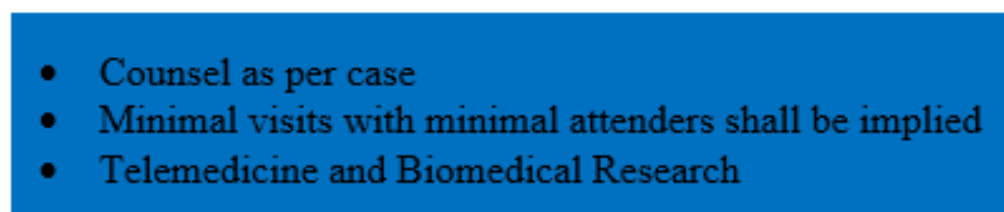

Figure 1: Stages of Orthopedic Practice care in Post-COVID-19 Era

The protocols issued by the Indian Orthopaedic Association (IOA) and various state associations of orthopedics need to be considered which provide the regulations and guidance for resuming the semi-elective and non-traumatic surgeries in the post-pandemic era. The protocol on safety measures and postoperative rehabilitation, telemedicine services, orthopedic 


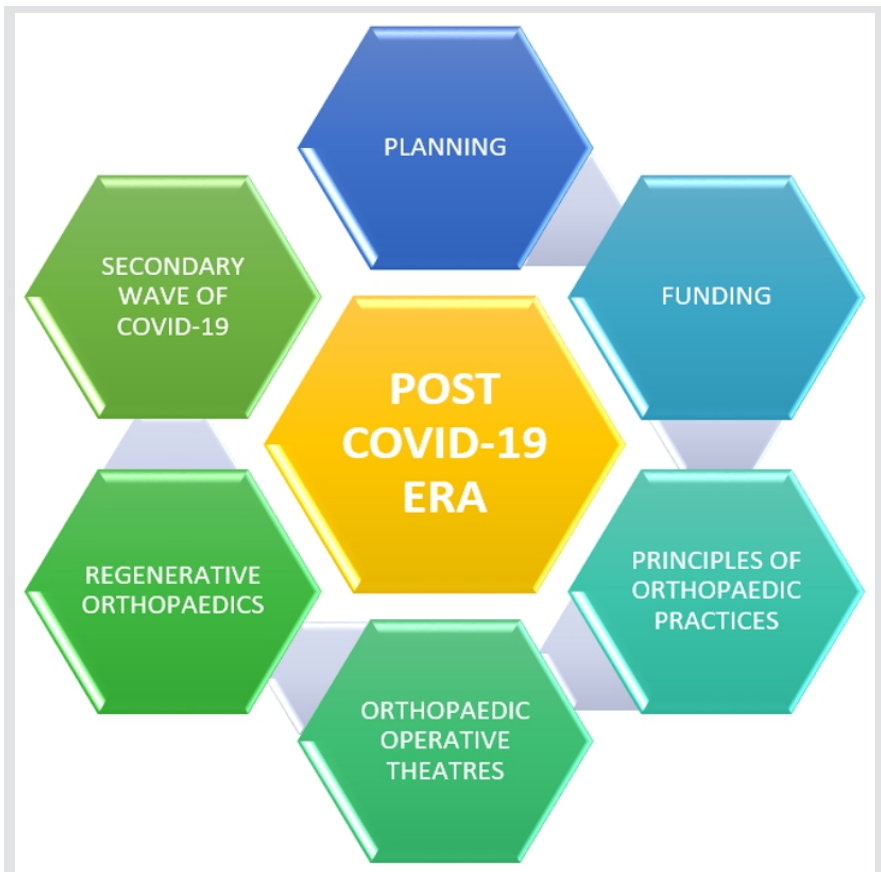

Figure 2: Stages of orthopedic practice in post-COVID-19 era.

education, and biomedical research has to strictly adhere on a long-term basis [7].

b) Funding

At present, the Government of India (GOI) has launched Pradhan Mantri Jan Arogya Yojana (AB-PMJAY) or Ayushman Bharat PM-JAY, which is the world's largest health insurance/assurance scheme fully financed by the GOI [8]. These health insurance policies serve approximately 50 million beneficiaries in secondary and tertiary hospitals across India. These schemes help patients who are unable to afford the treatment costs but still it's an additional financial burden to GOI in this lockdown period where the economic crisis prevails. Testing and treatment of COVID-19 are available for free under Ayushman Bharat, GOI provides free testing and treatment of COVID-19 patients. Orthopedic surgeons must understand the affordability and risk-benefit ratio in terms of planning treatment modality. The need of the hour is to prefer conservative methods over surgical interventions unless and until indicated.

c) Principles of Orthopedic Practices

Orthopedic practices in the post-COVID-19 era need to be

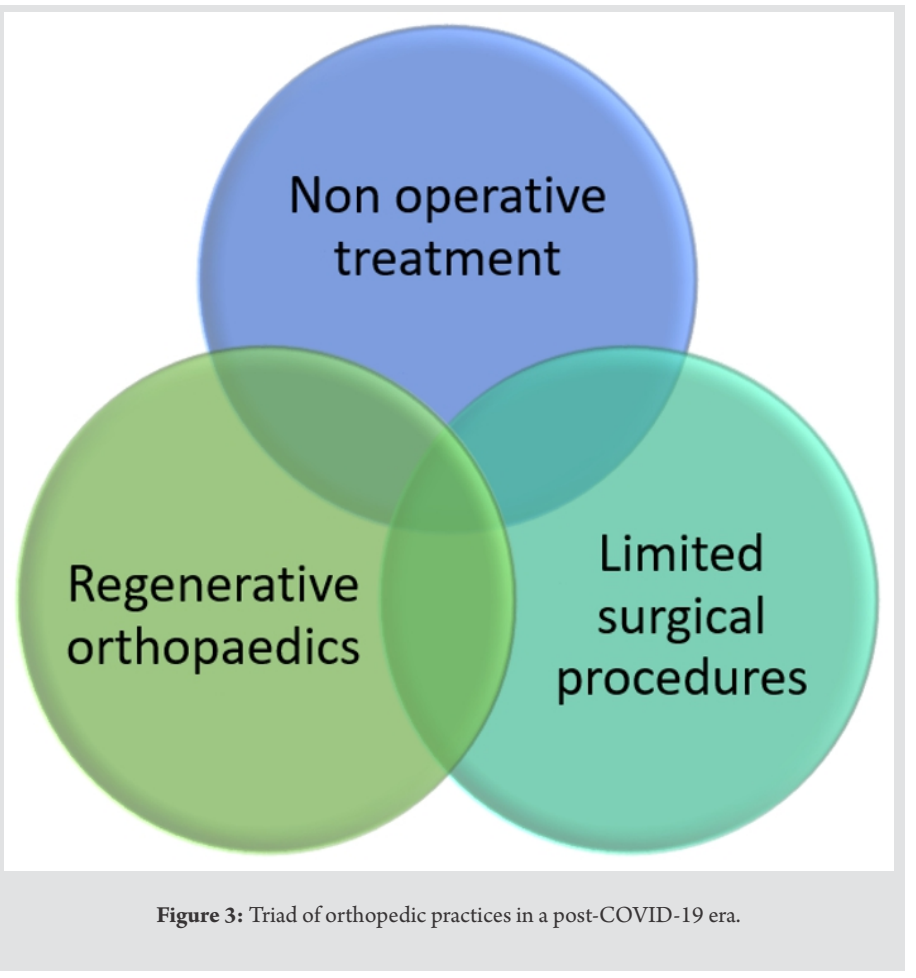

stratified meticulously. The art of non-operative management takes an utmost priority in the treatment pyramid. The triad of orthopedic practices in the post-COVID-19 era is depicted in (Fig. 3).

\section{i) COVID-19 testing}

All patients who are visiting OPD and emergencies have to be screened for COVID-19 by questionnaire method. The COVID19 suspects have to be screened by rapid card test and confirm by RT-PCR. The bag and baggage of patients and attenders have to be subjected to a hypochlorous acid shower for a minimum of 30 s. It is a mandate to the kith and kin of all patients to wear face masks and sanitizes their hands with $60-70 \%$ alcohol-based hand sanitizers. The motivation for interpersonal distancing has to be encouraged.

ii) Emergency services

The nature of injury and type of fracture should be analyzed. If the fracture tends to be unstable and needs fixation, a swab has to be sent for COVID-19 RT-PCR immediately before admission. In emergency OT, limit the exposure to the number of healthcare professionals and follow the principles of damage control orthopedics. All necessary precautions against COVID-19 have

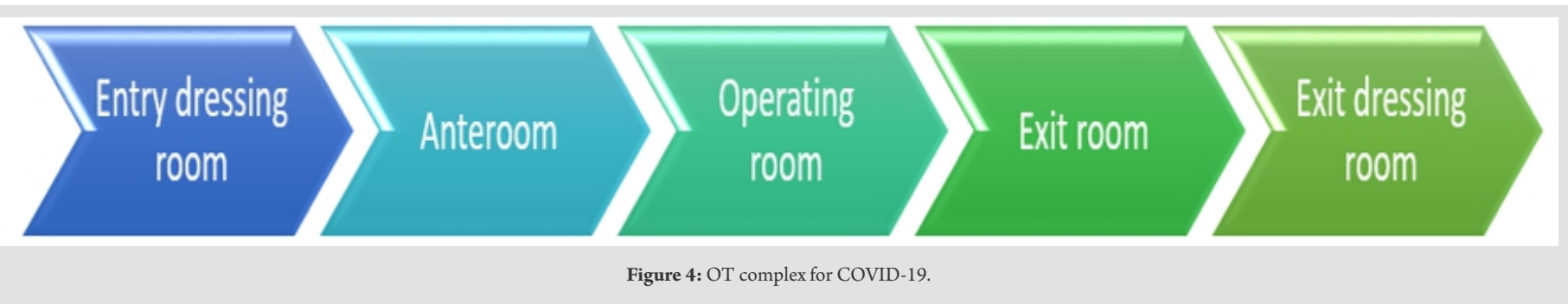

Journal of Orthopaedic Case Reports | Volume 11 | Issue 4 | April 2021 | Page 110-116 


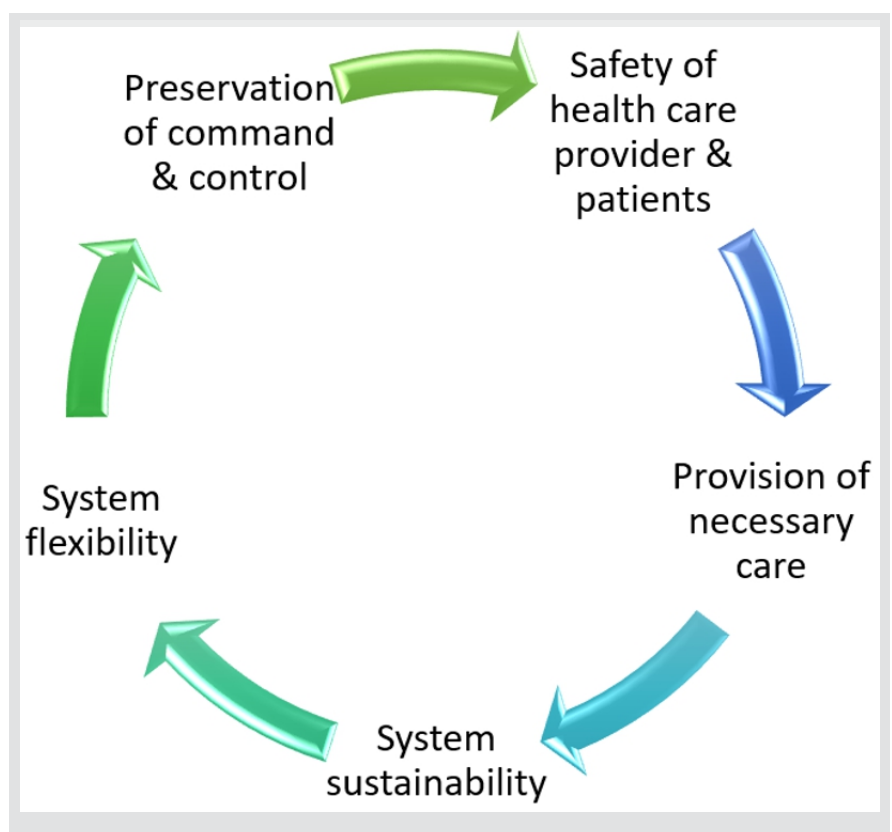

Figure 5: Structure of orthopedic residency program.

to be taken up by all health-care teams (in the form of hand washing, personal protective equipment [PPE] kits, and goggles) who are operating the patient.

iii) OPD services

A minimum number of attendees have to be entertained in the OPD chambers to avoid cross-infection. A robust resurgence of non-operative treatment of fractures would be the trend to balance the functional outcomes of the patients. Fractures have to be treated by non-operative methods in the form of slab, casts, brace, or tractions to achieve the biology of fracture union.

iv) IPD services

The minimum number of patients for postponed elective surgeries has to be admitted on a priority basis. In orthopedic wards, limit the entries of attenders. Minimum workforce both doctors and residents and allied health care workers have to be maintained in the wards. Due to international and national travel restrictions, economic recession, and unavailability of implants, the patient would be burdened more for surgical procedures. Once a COVID-19 patient gets operated on, the number of days of hospital stay has to be limited and utmost care to be taken for post-operative rehabilitation. The morbidity of surgery on COVID-19 patients has to be ascertained.

\section{v) Post-operative care}

Considering the hospital environment to be an infective one, the role of telemedicine and web assistance has to be ascertained for the benefit of patients. This ideology will lessen the frequency of hospital visits and face-to-face interaction with health-care professionals.

d) Orthopedic operation theaters

A separate OT complex must be dedicated for COVID-19 individuals for both emergencies and elective surgeries. Rodrigues-Pinto et al. suggested the dedicated OT for COVID19 into five rooms which are depicted in (Fig. 4) [9]. A separate "donning" and "doffing" rooms have to be identified and should be strictly followed.

Strict hand washing protocols have to be followed as per the surgical hand washing techniques. The OT must be fumigated before starting the positive COVID-19 case. The whole team who is inside the operating chambers has to be protected with PPE. The minimum number of surgeons, anesthetists, and staff nurses must be entertained inside the operating chambers in the OT. Orthopedic OT should be equipped with a HEPA filter and high-frequency laminar airflow system to reduce the maximal microbial contamination. Orthopedic OT needs special attention where aerosols would be generated more than expected as we would be using high-power drills and equipment. The practice of regional blocks and spinal anesthesia has to be followed by anesthetists. In unavoidable situations, general anesthesia has to be followed with all the necessary precautions. After the surgery of each positive COVID-19 case, the OT chamber has to be fumigated as per the norms. The instruments and implants used for that particular case have to be double sterilized. Importantly, the handling of biomedical waste has to be scrutinized in every step of transportation from wards to waste disposal area.

\section{e) Regenerative orthopedics}

During the pandemic, all fracture cases that have been managed conservatively have to be reviewed with follow-up radiographs. If any fracture case shows the signs of delayed or non-union, the application of regenerative orthopedics has to be considered to reduce the burden of orthopedic surgeons, to decrease the morbidity, and to improve the functional quality of life of patients.

In orthopedics, the trend of research is now focusing on preventive interventions and therapeutic solutions that can result in enhancing tissue regeneration either soft tissue or bone and reduction of degenerative mechanisms [10]. The recent ideology of "Orthobiologics" has led to the development of less invasive procedures and accelerated treatments which enhance the functional recovery of musculoskeletal disorders. Percutaneous administration of substances with osteoinductive and osteogenic properties offers the advantage of decreased morbidity when compared to classic techniques [11].

At present, the continuous scrutinization of bioactive micromolecules has led to the discovery of minimally invasive techniques to combat patients with musculoskeletal disorders. These bioactive molecules bridge a gap between conservative and surgical management in the treatment of orthopedic disorders. In this context, platelet-rich plasma is a potent orthobiologic that acts as a biological enhancer and resorts as a 
minimally invasive procedure for treating all soft-tissue disorders and fracture management in cases of delayed or non-union [12]. Mesenchymal stem cells (MSCs) produced a major glitch in the orthopedic community and showed a positive response in various disorders such as spinal cord injury, nerve disorders, osteoarthritis, avascular necrosis, delayed and non-union of fractures, and so on. MSCs paved a better way for the cases with severe morbidity and showed a ray of hope for certain orthopedic disorders [13]. The knowledge about regenerative orthopedics has to be disseminated and encourage its use among orthopedic surgeons in various disorders.

We recommend that the audit of all cases that have been taken treatment with regenerative orthopedics has to be well maintained for assessing the functional outcome, to compare the treatment efficacy with classical techniques, and for further research.

\section{f) Secondary wave of COVID-19}

It is very well documented in the literature that the occurrence of pandemic weans off with multiple secondary waves of the pandemic disease. The occurrence of secondary waves after SARS, Spanish flu, and dengue warns the probability of the occurrence of COVID-19 secondary waves. Hence, an orthopedic surgeon must forecast and predict the severity of secondary waves of the COVID-19 pandemic and follow a paradigm shift in the practice of orthopedic surgery in the community $[14,15]$. We should follow the strict protocols of handling a pandemic situation for the secondary wave as well. One should anticipate the mitigation of the secondary wave and be prepared with a disaster management team ready to win the battle of secondary waves during the pandemic.

\section{Telemedicine}

The literal meaning of telemedicine is healing at a distance. It is a broad term used to encompass health-care delivery and other activities such as education, research, and public health promotion [16]. As per American Telemedicine Association, "Telemedicine is the natural evolution of health care in the digital world" [17].

According to the WHO, telemedicine is "the delivery of healthcare services, where distance is the critical factor, by all health-care professionals using information and communication technologies for the exchange of valid information for the diagnosis, treatment, and prevention of disease and injuries, research and evaluation, and for continuing education of healthcare providers, all in the interests of advancing the health of individuals and their communities" [18]. Although telemedicine is not so popular in India, various projects such as ICMRAROGYASREE [19], National e-health authority [20], and Village Resource Center [21] paved their way to success after 2005 when National Telemedicine Taskforce was set by Health Ministry of India.
The nationwide lockdown imposed by the GOI to combat the spread of COVID-19 and fear among the people of getting infected while visiting health-care centers have resorted to consider the practice of telemedicine. It is believed that telemedicine can be vital for outpatient care during and postpandemic. In orthopedic practice, follow-up is very critical in terms of post-operative outcome and rehabilitation program [22]. Lamminen et al. in 1996 found that telemedicine is viable for orthopedic consultation [23]. Clinical outcome is assessed after 1 year in $80-95 \%$ of trauma patients [24].

Amidst the COVID-19 pandemic, it is very difficult for patients to visit outpatient departments for follow-up. In this age, as most of the rural and the urban population in India have access to smartphone and internet services, thereby telemedicine may be the solution for remote follow-up during this pandemic. In the post-pandemic era, the challenges may be manifold and our response should be adequate. Health experts and researchers must put their ideas together to design a proper strategy and future policy based on the experiences during the pandemic and challenges to be faced post-pandemic era. Short educational videos should be shown for post-operative rehabilitation purposes to demonstrate various exercises during follow-up care.

\section{Orthopedic Education}

In wake of the COVID-19 pandemic, it is not much clear about the impact on medical education. Medical faculty and students are already grappling with the changes made during the pandemic and are trying to accommodate these changes in the plan of their career development. Although online equivalents to in-person teaching are the necessity at this time of pandemic, it has some drawbacks such as loss of collaborative experiences that may have a significant detrimental effect on education. The seriousness of the prevailing situation has led to the cessation of bedside teaching which is essential for the development of clinical skills and rapport formation. Notably, this constitutes another set of issue which needs to be resolved at the earliest.

One unique way to resolve this issue is to implement technology. One of the immediate changes introduced during pandemic has been the replacement of in-person classes by recorded lectures or live streams. Now, classes have been moved online, standards of these interactive discussions online need to be studied to ensure that the online experience is adequate. This will be a challenge to reengage medical students once restrictions are lifted. The orthopedic residency program structure was proposed by Schwartz et al. which is depicted in (Fig. 5) [25].

Practicing interpersonal distancing also precludes residents from attending studios, lecture halls, and small group rooms. Further due to cessation of outpatient services, not admitting the nonemergency patients, allocating orthopedic wards for COVID-19 patients, and posting orthopedic residents in COVID-19 duties to combat the shortage of workforce have added to the effect. 
Inability to attend conferences and presentations, many medical students lost the opportunity for personal development. The orthopedic research work is also badly affected due to the pandemic as it is largely based on the follow-up of trauma patients.

Educational experts and researchers must come with forward thinking amidst this pandemic and based on their experience to design the scholarly approach to solve this issue. The COVID-19 pandemic may bring the transformation in the field of medical education and training with the advancement of telehealth, adoption of new research protocols, and flexible approaches for clinical trials. Medical students and educators can help to document and analyze the challenges and effects of the pandemic which can be applied to lay down new principles and practices in the future.

\section{Recommendations by Health Organizations}

The WHO [2], MOHFW [5], ICMR [6], IOA [7], and public health-care experts opine on the protective measures as follows:

- Sanitization of bag and baggage of the patients with hypochlorous acid shower exposure for at least $30 \mathrm{~s}$ while entering and leaving the hospital

- Temperature screening by infrared thermometer

- Mandatory wearing of face mask either surgical or N-95 mask by all persons who enter the hospital

- Maintenance of interpersonal distancing among patients, attenders, and health-care professionals

- Implementation of rapid card test to check the immunity status of COVID-19

- Mandatory COVID-19 testing for both the patients with symptoms and the suspects without symptoms

- Implementation of alcohol-based hand sanitizer

- Scheduled elective surgical procedures to be postponed till pandemic wave flattens

- Minimal use of ICUbeds, PPE, and ventilators

- Introduction of regenerative orthopedics, minimally invasive surgeries, telemedicine, and biomedical research
- Disaster preparedness for the secondary wave of COVID19.

\section{Conclusion}

COVID-19 has rapidly evolved, precipitated, and inconceivably disrupted our health-care system. It poses a significant psychological burden on every health-care provider in this period. A proper protocol to rationalize the working hours, safety precautions, departmentalization, and resource allocation to combat the aftermath of the COVID-19 pandemic is mandatory. Indeed, this will ensure better functioning of the department which, in turn, will enhance the psychological resilience among the health-care professionals. The resumption of surgical management should be advocated in a step-wise manner. Albeit pandemic has withheld bedside teachings and designed medical curriculum, however, the advanced technology has made it plausible to impart knowledge regarding orthopedic education and research as part of postgraduation residential programs. This shall be liable to continue as a major mode for propagating medical education in the post-pandemic phase and thereby warrants equivalent attention under the planning section for the same. The relegation of this pandemic and substantially mitigating the secondary waves compels for interdisciplinary research work to have an amicable assortment. With the rollout of vaccines in the country, the scenario is in favor of returning to normalcy with evaluation for COVID-19 being added to the list of routine medical and surgical screening profiles.

\section{Ethical Approval}

This article does not contain any studies with human participants or animals performed by any of the authors.

\section{Clinical Message}

Tough time calls for tougher decisions in life and in health care. This article helps the readers on the advisory guidelines and overall management algorithms on the various spectrum of orthopaedic care during COVID-19 pandemic crisis .

\section{References}

1. Huang C, Wang Y, Li X, Ren L, Zhao J, Hu Y, et al. Clinical features of patients infected with 2019 novel Coronavirus in Wuhan, China. Lancet 2020;395:497-506.

2. World Health Organization. Coronavirus Disease 2019 (COVID-19) Situation Report No. 51;2020.

3. Chhabra HS, Bagaraia V, Keny S, Kalidindi KK, Mallepally A, Dhillon MS. COVID 19: Current knowledge and best practices for orthopaedic surgeons. Indian J Orthop 2020;54:411-25.
4. Centers for Disease Control and Prevention, Novel Coronavirus (2019-nCoV) Situation Summary; 2019. Available from: https://www.cdc.gov/coronavirus/2019ncov/casesupdates/summary. [Last accessed on $2021 \mathrm{Apr}$ 27].

5. Ministry of Health and Family Welfare (MOHFW); 2021. Available from: https://www.mohfw.gov.in/pdf / AdvisoryforHospitalsandMedicalInstitutions.pdf. [Last accessed on 2021 Apr 27]. 
6. Indian Council of medical Research SARS-CoV2(COVID-19) Testing; 2020. Available from: https:// www.icmr.nic.in/content/covid-19. [Last accessed on 2021 Apr 27].

7. IOA Updated Advisory during COVID-19; 2021. Available from: https:// www.ioaindia.org. [Last accessed on 2021 Apr 27].

8. Pradhan Mantri Jan Arogya Yojana; 2021. Available from: https://www.pmjay.gov.in/about/pmjay. [Last accessed on 2021 Apr 27].

9. Rodrigues-Pinto R, Sousa R, Oliveira A. Preparing to perform trauma and orthopaedic surgery on patients with COVID-19.J Bone Joint Surg Am 2020; 102:946-50.

10. Simpson AH, Mills L, Noble B. The role of growth factors and related agents in accelerating fracture healing. J Bone Joint Surg Br 2006;88:701-5.

11. Wilkins RM, Chimenti BT, Rafkin RM. Percutaneous treatment of long bone non-unions: The use of autologous bone marrow and allograft bone matrix. Orthopaedics 2003;26:549-54.

12. Ramesh R, Jeyaraman M, Prajwal GS. Autologous platelet rich plasma therapy in orthopaedics: An update. Natl J Clin Orthop 2018;2:33-6.

13. Ramesh R, Jeyaraman M, Chaudhari K, Prajwal GS, Dhamsania HJ. Mesenchymal stem cells-a boon to orthopaedics. Open J Regen Med 2018;7:19-27.

14. Strzelecki A. The second worldwide wave of interest in coronavirus since the COVID-19 outbreaks in South Korea, Italy and Iran: A Google trends study. Brain Behav Immun 2020;88:950-1.

15. Cyranoski D. We need to be alert: Scientists fear second coronavirus wave as China's lockdowns ease. Nature 2020. (Ahead of print)

16. Wilson LS, Maeder AJ. Recent directions in telemedicine: Review of trends in research and practice. Healthc Inform Res 2015;21:213-22.
17. Home-ATA Main; 2021. Available from: http:// www.americantelemed.org/ home. [Last accessed on 2021 Apr 27].

18. World Health Organization. Telemedicine-Opportunities and Developments in Member States. 2nd ed. Geneva, Switzerland: World Health Organization Press; 2010. Available from: https://www.who.int /goe/publications/ goe_telemedicine_2010.pdf. [Last accessed on 2021 Apr 27].

19. ICMR Project; 2018. Available from: http:// www.dos.iitm.ac.in/ projects/ icmr. [Last accessed on 2021 Apr 27].

20. National eHealth Authority (NeHA), National Health Portal of India 2021;2021. Available from: https://www.nhp.gov.in/national_ehealth_authority_ne ha_mtl. [Last accessed on 2021 Apr 27].

21. Mishra SK, Singh IP, Chand RD. Current status of telemedicine network in India and future perspective. Proc Asia-PacAdv Netw2012;32:151-63.

22. Harle D, Ilyas S, Darrah C, Tucker K, Donell S. Community-based orthopaedic follow-up. Is it what doctors and patients want? Ann R Coll Surg Engl 2009;91:66-70.

23. Lamminen H, Nevalainen J, Alho A, Lindholm TS, Tallroth K, Lepistö J. Experimental telemedicine in orthopaedics. J Telemed Telecare 1996;2:170-3.

24. Castillo RC, Mackenzie EJ, Bosse MJ, LEAP Study Group. Orthopaedic trauma clinical research: Is 2-year follow-up necessary? Results from a longitudinal study of severe lower extremity trauma.J Trauma 2011;71:1726-31.

25. Schwartz AM, Wilson JM, Boden SD, Moore TJ, Bradbury TL, Fletcher ND. Managing resident workforce and education during the COVID-19 pandemic: Evolving strategies and lessons learned. JB JS Open Access 2020;5:e0045.

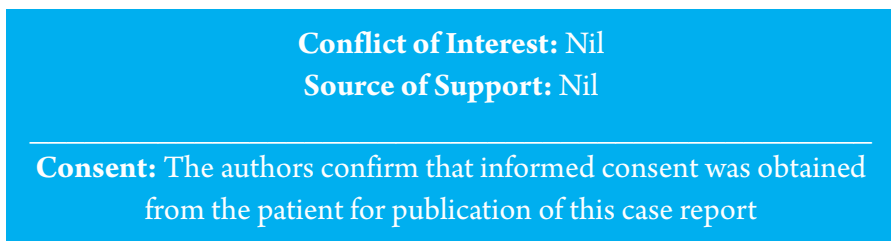

\section{How to Cite this Article}

Jeyaraman M, Muthu S, Shyam A. Guest Editorial- Indian Paradigm of Orthopedic Care in Post-COVID-19 Era. Journal of Orthopaedic Case Reports 2021 April 2021;11(4):Page 110116. 